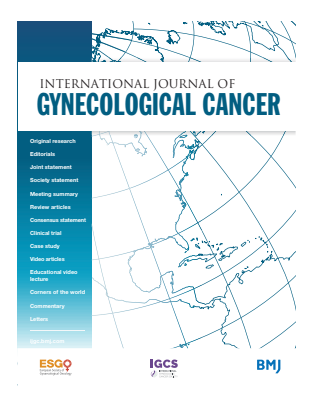

${ }^{1}$ Gynecology Oncology Unit, Hospital Universitario de Donostia, San Sebastian, Spain ${ }^{2}$ Gynecology and Obstetrics Department, Hospital Universitario Donostia, San Sebastian, Spain

${ }^{3}$ Obstetrics and Gynecology, Hospital Universitario 12 de Octubre, Madrid, Madrid, Spain

\section{Correspondence to}

Dr Ibon Jaunarena, Gynecology Oncology Unit, Hospital Universitario de Donostia, San Sebastian 20014, Spain; IBON.JAUNARENAMARIN@ osakidetza.eus

Accepted 19 May 2020 Published Online First 21 June 2020

\title{
Laparoscopic complete resection of bulky precaval nodes by extraperitoneal approach in a patient with advanced endometrial cancer
}

Ibon Jaunarena (D) ,' Mikel Gorostidi (D) ,2 Ruben Ruiz, ${ }^{2}$ Cecilia Villalain (D) , ${ }^{3}$ Paloma Cobas, ${ }^{2}$ Arantza Lekuona ${ }^{2}$

In advanced endometrial cancers, the benefit in survival of cytoreductive surgery, imitating advanced ovarian cancer, has been suggested (Havrilesky 2015). ${ }^{1}$ In stage IIIC the size of the lymph nodes dictate the possibility of resection and this challenging surgical effort is often performed by laparotomy, which is still the standard of care.

This video describes step-by-step the complete removal of precaval bulky nodes by the extraperitoneal laparoscopic approach. It shows the benefits and feasibility of this technique, given its high definition and technical accuracy.

This technique allows horizontal access to the cleavage plane, minimizing the risk of injury to the great vessels and bleeding, whereas a transperitoneal access requires a more vertical approach to the dissection plane, and therefore poses a greater risk of injury.

A 49-year-old woman was referred for incidental endometrial cancer after hysterectomy. A computed tomography scan revealed a $3.5 \times 2.5 \mathrm{~cm}$ sized endometrioid carcinoma invading $>50 \%$ of the myometrium with lymphovascular invasion and suspicion of metastasis in the pelvic and para-aortic lymph nodes. Thus, following the European Society of Gynaecological Oncology (ESGO) guidelines, surgical staging and debulking was planned using a minimally invasive technique.

After extraperitoneal para-aortic space exposure, enlarged pelvic, left aortic and precaval lymph nodes were confirmed. A primary complete laparoscopic

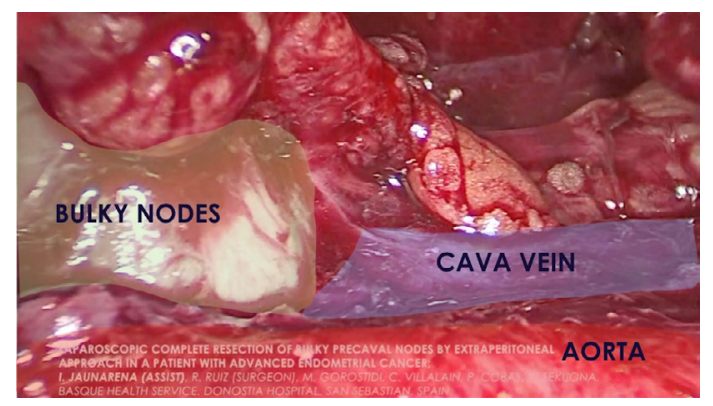

Figure 1

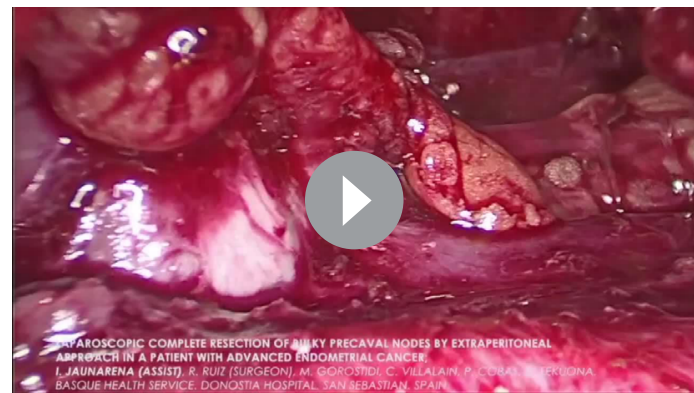

Video 1 Bulky nodes above the vena cava and right ureter.

lymphadenectomy was performed up to the left renal vein using a harmonic sealing device. The right ureter was firmly attached to the inframesenteric precaval $2-3 \mathrm{~cm}$ bulky nodes (Figure 1: bulky node above the vena cava and right ureter). The ureter was released, and the resection of the bulky nodes was accomplished by careful blunt dissection.

The surgery also comprised other transperitoneal procedures, which are not included in this video (pelvic lymph node debulking and oophorectomy) and lasted 465 min. Povidone-iodine was applied to the trocars. The surgery was carried out in Donostia Hospital (Spain) by a senior oncological surgeon and a fellow. The final lymph node biopsy confirmed macrometastasis in the pelvic and paraaortic nodes (International Federation of Gynecology andObstetrics (FIGO) IIIC2). All oncological security measures have since been carried out to prevent tumor spread.

Complete laparoscopic resection of bulky precaval nodes in advanced endometrial cancer is feasible and provides excellent accuracy in trained hands.

\section{Twitter Mikel Gorostidi @mgorostidi}

Contributors IJ was the surgeon assistant, edited the video and prepared the manuscript. MG prepared the manuscript, did the narration, prepared all the slides and edited the video. RR was the surgeon and helped in the preparation and correction of the final manuscript. CV prepared the narration and corrected the manuscript. PC and AL contributed in the preparation of the manuscript. 
Video article

Funding The authors have not declared a specific grant for this research from any funding agency in the public, commercial or not-for-profit sectors.

Competing interests None declared.

Patient consent for publication Not required.

Ethics approval The study was approved by our Institutional Review Board.

Provenance and peer review Not commissioned; externally peer reviewed.

Data availability statement All data relevant to the study are included in the article
ORCID iDs

Ibon Jaunarena http://orcid.org/0000-0003-2081-7862

Mikel Gorostidi http://orcid.org/0000-0001-5150-2797

Cecilia Villalain http://orcid.org/0000-0002-9456-4100

\section{REFERENCE}

1 Havrilesky LJ, Cragun JM, Calingaert B, et al. Resection of lymph node metastases influences survival in stage IIIC endometrial cancer. Gynecol Oncol 2005;99:689-95. 